
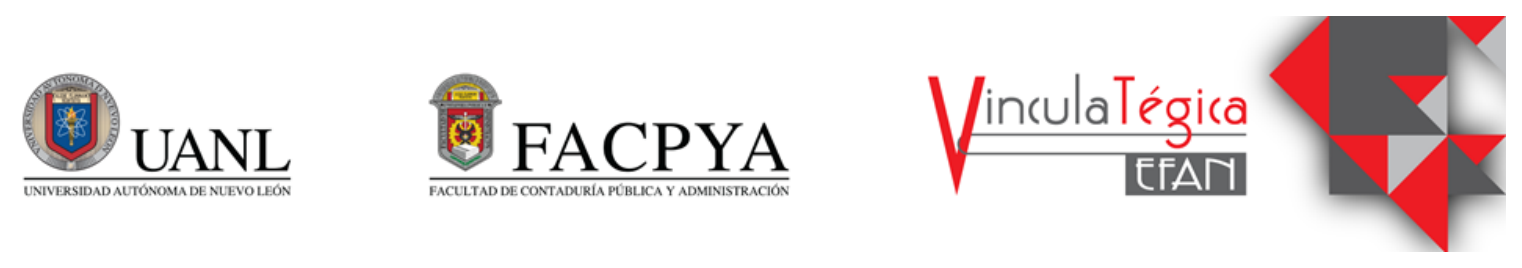

\title{
Una metodología para la gestión del conocimiento basada en arquitectura de procesos para una PyME constructora (diseño preliminar)
}

\author{
Ruth María Zepeda Estrada ${ }^{1}$, Alonso Pérez Soltero ${ }^{2}$ y Mery Pesantes Espinoza ${ }^{3}$ \\ ${ }^{1}$ Universidad de Sonora, ruth_ze@hotmail.com, Blvd. Luis Encinas y Rosales S/N, Col. Centro Hermosillo, \\ Sonora, México, (662) 2592159 \\ ${ }^{2}$ Universidad de Sonora, aperez@industrial.uson.mx, Blvd. Luis Encinas y Rosales S/N, Col. Centro \\ Hermosillo, Sonora, México, (662) 2592159 \\ ${ }^{3}$ Universidad de Sonora, mery.pesantes@unison.mx, Blvd. Luis Encinas y Rosales S/N, Col. Centro \\ Hermosillo, Sonora, México, (662) 2592159
}

Información del artículo revisado por pares

Fecha de aceptación: junio-2021

Fecha de publicación en línea: diciembre-2021

DOI: https://doi.org/10.29105/vtga7.1-128

\begin{abstract}
Resumen
En este artículo se presenta un diseño preliminar de una metodología para la gestión del conocimiento (GC) de una empresa pequeña y mediana (PyME) cuyo giro de negocio es la construcción a través de la implementación de una arquitectura de procesos (AP). En esta empresa se puede observar una falta de control general de sus procesos desde su diseño hasta su evolución, falta de documentación de sus procesos, falta de manejo de toda la información y el conocimiento contenido en sus procesos, y una falta de mecanismos para compartir conocimiento entre roles de sus procesos. La AP ha sido reconocida como un medio para gestionar de forma global un sistema de procesos, lo que permitirá resolver problemas anteriormente mencionados. $\mathrm{La}$ metodología se estructuró utilizando de base 2 enfoques de gestión: procesos y conocimiento, las cuales se ejecutan en sincronía a lo largo de toda la metodología. Esta consta de 4 fases: 1) entender el contexto de la organización e identificar el conocimiento existente en ella; 2) diseñar una AP y crear herramientas para transferir y almacenar el conocimiento; 3) construir y ejecutar la AP y aplicar los conocimientos; y 4) mantener la AP y actualizar el conocimiento de la organización. Finalmente, esta metodología busca apoyar a las PYMEs a tener un mejor control de sus procesos de negocio, a identificar, reutilizar y compartir conocimiento de
\end{abstract}

Abstract
This article presents a preliminary design of a methodology for knowledge management (KM) of a small and medium-sized company (SME) whose business line is construction through the implementation of a process architecture (PA). In this company it is possible to observe a lack of general control of its processes from its design to its evolution, a lack of documentation of its processes, a lack of management of all the information and knowledge contained in its processes, and a lack of mechanisms for sharing inter-role knowledge of your processes. The AP has been recognized as a means to globally manage a system of processes, which will allow solving the aforementioned problems. The methodology was structured using two basic management approaches: processes and knowledge, which are executed in synchrony throughout the entire methodology. This consists of 4 phases: 1) understand the context of the organization and identify the existing knowledge in it; 2) design a PA and create tools to transfer and store knowledge; 3) build and run the PA and apply knowledge; and 4) maintain the PA and update knowledge of the organization. Finally, this methodology seeks to support SMEs to have better control of their business processes, to identify, reuse and share knowledge of administrative and technical activities carried out by process roles, in addition to facilitating changes due 
actividades administrativas y técnicas llevadas a cabo por roles de procesos, además de facilitar la realización de cambios debido a su entorno de trabajo dinámico.

Palabras clave: Arquitectura de procesos, Gestión del conocimiento, Gestión de la innovación (O32), Gestión de la tecnología de la información (M15), Gestión laboral (M54)

\section{INTRODUCCIÓN}

Este proyecto surge en una empresa constructora con más de 30 años operando, en donde el personal estratégico y con más experiencia, que son los socios, están considerando retirarse, pero al no contar con mecanismos de control que les permitan continuar operando de la misma forma en que se ha hecho siempre, sin necesidad de estar presentes pero con la certeza de que se lleven a cabo correctamente cada uno de los procesos que intervienen en sus operaciones, no les es posible retirarse y conservar su empresa.

Además, en ella existe mucha desorganización y desorden, debido a que las actividades, tanto administrativas como operativas, se van realizando conforme van surgiendo necesidades, de la misma forma se asignan responsabilidades conforme avanzan los proyectos y de acuerdo a lo que se va requiriendo. El control lo lleva cada persona por separado y estas no saben específicamente con qué información cuenta cada quien ni quien la tiene.

La empresa tampoco cuenta con una descripción ni estructura de los puestos de trabajo, son la propia experiencia y el dominio de actividades por parte de cada integrante de la organización, lo que hace que la empresa pueda seguir su curso aparentemente sin ningún problema, sin embargo, existe mucho descontrol y se pierde mucho tiempo al momento de buscar información y/o conocimiento específico requerido. Los socios llevan el control en cuadernos y solo utilizan herramientas tecnológicas para ciertas cosas y a través de otras personas, por lo que gastan el doble de tiempo en ello, lo cual provoca que la información se pierda to their dynamic work environment.

Keywords: Process architecture, Knowledge management, Innovation management (O32), Information technology management (M15), Labor management (M54).

fácilmente o sea difícil de encontrar.

Para resolver la problemática anterior, el objetivo de este artículo es mostrar el diseño preliminar de una metodología basada en una arquitectura de procesos que además de gestionar los procesos le permita a la organización gestionar también su conocimiento.

El artículo está estructurado en cinco secciones incluyendo esta introducción, luego un marco teórico es mostrado donde se exponen los conceptos más relevantes y las metodologías utilizadas como base para el desarrollo de esta propuesta. A continuación, se describe el método de elaboración de la propuesta metodológica seguido del resultado, un diseño preliminar de la metodología propuesta con la descripción detallada de cada una de sus fases. Finalmente, se muestran las conclusiones del trabajo y las referencias consultadas para el desarrollo del mismo.

\section{MARCO TEÓRICO}

A continuación se presentan los conceptos más importantes e investigaciones previas para este trabajo:

\subsection{PYMEs constructoras}

Son organizaciones intensivas en conocimiento y altamente dependientes de las competencias de sus profesionales (Kazi, 2005). Existen 3 características dentro de la construcción, en donde la gestión del conocimiento (GC) juega un papel importante: el primero, en la constructabilidad, interesado en la calidad y en el uso óptimo del conocimiento para lograr mayor productividad, utilizando como estrategia la coordinación de funciones, espacios y tiempo 
de las distintas especialidades que se involucran dentro de un proyecto (Hartman y Fisher, 2007); el segundo, en la mejora de productos y procesos, enfocada en simplificar el trabajo, eliminar errores y en aumentar la productividad, utilizando como estrategia principal el aprendizaje generado por la experiencia, así como el ejercicio del ensayo y error (Kivrak, Gokhan, Dikmen y Birgonul, 2008); y el tercero, en la innovación, que se enfoca en la integración de nuevos materiales, procesos y tecnologías, ya sea imitando tendencias, adaptándose a cambios e incluso desarrollando nuevas formas de hacer las cosas (Chinowsky y Carrillo, 2007).

El enfoque en los procesos es una de las propuestas de GC para empresas constructoras (Shelbourn,et al., 2006), a su vez, una de las tres dimensiones clave en GC, es la organizacional, interesada en la estructura adoptada de acuerdo al proceso productivo y a los roles y responsabilidades asignadas (Schwartz, 2006). El conocimiento es un activo que juega un papel decisivo en la eficiencia del rendimiento y la eficiencia de la adaptación de los procesos de las organizaciones (Szelagowski y BerniakWozny, 2019), por lo que las empresas buscan múltiples alternativas para aumentar el conocimiento mediante el modelamiento de la información generada en los procesos del negocio (Portela, et al., 2014).

\subsection{Gestión del Conocimiento}

Se define como la capacidad de administrar eficazmente los flujos de conocimiento al interior de la organización para garantizar su acceso y reutilización permanente (Angulo, 2017), con lo cual se pueden generar cambios $y$ resultados sustentables, optimizar recursos, aprovechar el conocimiento, aprender permanentemente, disminuir costos, aumentar la creatividad e innovación, aumentar el rendimiento e ingresos por ventas, así como el mejoramiento organizacional (Marulanda, Giraldo y Serna, 2015). La GC para Choo (1998) aparece como una solución que permite administrar lo que la organización sabe, integra a las personas, la tecnología, los procesos y la estructura de una organización.
El ciclo de vida de GC se expone en múltiples modelos entre ellos los de Meyer y Zack (1996), Bukowitz y Williams (2000), Mcelroy (1999), Wiig (1993), Carlile y Rebentisch (2015) y Heisig (2009), los cuales sintetizó Dalkir (2017). La determinación de siete etapas principales presentadas por Castillo, Farías, Lavín y Pedraza (2015) quedó de la siguiente manera: identificación, donde se reconocen la fuentes de conocimiento de la empresa; creación, donde se generan nuevas ideas que sirven para dar soluciones e innovar; transferencia, que es la forma en la que se promueve la comunicación y disponibilidad del conocimiento; almacenamiento, donde se resguarda el conocimiento; la aplicación, donde se utiliza el conocimiento y por último la evaluación para conocer el comportamiento $\mathrm{y}$ el valor que genera el conocimiento a la organización.

\subsection{Arquitectura de Procesos}

Es definida como un marco conceptual para diseñar y mantener procesos y sus relaciones, el cual debe estar alineado con la estrategia y objetivos de negocio y la arquitectura empresarial (Pesantes, Lemus, Mitre y Mejía, 2012).

La AP consiste en un mapeo que ayuda a visualizar las relaciones de un proceso con otro y procesos futuros de una organización, describir sus interdependencias y otras relaciones entre sus elementos y procesos externos (Harmon, 2003), al comprender los procesos de trabajo y cómo documentarlos se puede gestionar el conocimiento de la organización (Omotayo, 2015).

El ciclo de vida de AP expuesto por Pesantes y Perez-Soltero (2020) presenta un proceso que contiene 7 meta-actividades que aparecen en el orden que deben ser llevadas a cabo, las cuales son: Compromiso, dónde se muestra la contribución que puede tener realizar la AP en la empresa, se obtiene patrocinio por parte de la gerencia y se identifican las partes interesadas así como las necesidades de comunicación. Requerimientos, donde se identifican y 
documentan los requisitos que debe tener la AP para lograr la mejora de procesos, qué nivel de detalle deben tener los procesos, qué impacto se tendrá, las limitaciones de la empresa, los atributos que facilitarán la actualización o expansión de los procesos y los objetivos comerciales a los cuales debe ser alineada la AP, en esta parte se debe entender el contexto de los procesos de la organización. Diseño, donde se analizan alternativas estructurales e identifican soluciones, se diseña el proceso, sus elementos e interfaces, se especifican lineamientos y la estandarización de los procesos y se seleccionan las herramientas para diseñar la AP. En la meta-actividad de construcción se recolectan y catalogan los procesos y sus elementos, se agrupan en vistas, se realiza el mapeo y el desarrollo de los procesos estándar, así como los ajustes necesarios. La siguiente meta-actividad del ciclo de vida AP es la validación, dónde se define como será evaluada y medida la AP para verificar que cumpla con los requisitos. Despliegue, es la meta-actividad donde se realiza la implementación de AP y mantenimiento, la última meta-actividad donde se realiza el monitoreo y se establecen mecanismos, herramientas y métodos para realizar cambios a la AP.

\subsection{Metodologías AP.}

La metodología BPTrends (del inglés, BPTrends Process Change Methodology) de Harmon (2014) es un enfoque para obtener una arquitectura de procesos. Abarca dos métodos complementarios: uno para el desarrollo de la arquitectura de procesos de negocios y otro para proyectos de rediseño de procesos de negocio.

El método AP considera en su primera fase entender a la organización y su contexto como un todo, se definen cadenas de valor, solidificando así el conocimiento sobre los objetivos y metas de cada una de ellas.

La segunda fase se enfoca en la definición de la arquitectura de procesos de las cadenas de valor definidas en la primera fase. Tanto los recursos, como las políticas y reglas de negocio, los recursos tecnológicos, los recursos humanos, entre otros, se adecuan a los procesos.

En la tercera fase se define cómo se realizará el monitoreo y la medición del proceso; en esta fase se requieren establecer indicadores clave de rendimiento, mediante estos indicadores se comprueba que los objetivos, estrategias e iniciativas relacionadas se están logrando.

En la cuarta fase se establece la gobernanza del proceso para que todos los procesos tengan controles comunes.

Por último, la quinta fase donde se alinean las capacidades empresariales para sincronizar todos los esfuerzos de las unidades de los procesos hacia una misma meta, llevando a cabo esta metodología continuamente se logran gestionar los procesos empresariales. La figura 1 muestra dicha metodología.

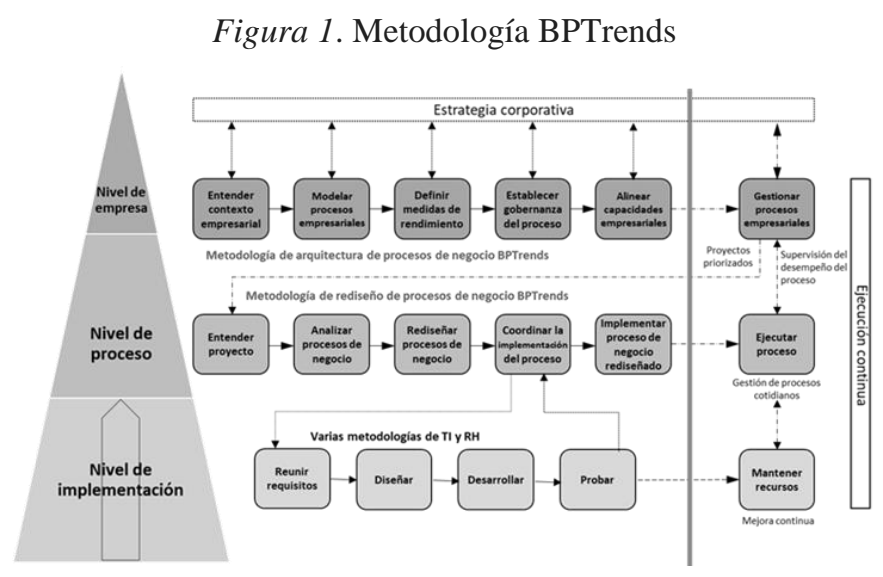


Existe una metodología desarrollada por Gómez y Luna (2013), donde la AP se basa en un modelo de competencias basado en GC. En él se desarrollan cuatro etapas secuenciales. La primera es "planear", donde se realiza un proceso de análisis del direccionamiento estratégico, el análisis de la misión y visión de la empresa, así como el de las debilidades, fortalezas, amenazas y oportunidades, también se analizan las áreas estratégicas, los objetivos y actividades, y se realiza la recopilación, estructuración, validación y presentación de toda la información. En la segunda etapa, llamada "hacer" se lleva a cabo un proceso de caracterización y estructuración de cargos y funciones, así como la definición y establecimiento de las competencias tanto específicas como organizacionales. En la etapa de verificación, se diseñan los perfiles de cargo de las competencias y se evalúan para identificar indicadores que reflejen su cumplimiento. Por último en la etapa de "actuar", se implementa el modelo, para lo cual se realizan procesos de sensibilización, capacitación y de mejora continua, además en esta etapa también se verifican los resultados obtenidos con el modelo y se replantean objetivos.

La referencia inicial de procesos es el ciclo PHVA, este a su vez se despliega en procesos y así de manera cada vez más específica de los diferentes procesos se pasa a los subprocesos. Posteriormente, de los procesos se plantean los objetivos que se desean alcanzar, para lo cual se determinan actividades con sus respectivas entradas y salidas, así como las personas responsables para cada caso. Seguido de esto se identifican las actividades y las tareas de mediano y corto plazo que se deben realizar para completar el proceso (Gómez y Luna, 2013). En la figura 2 se muestra la metodología expuesta.

Figura 2. Metodología AP de modelo de competencias.

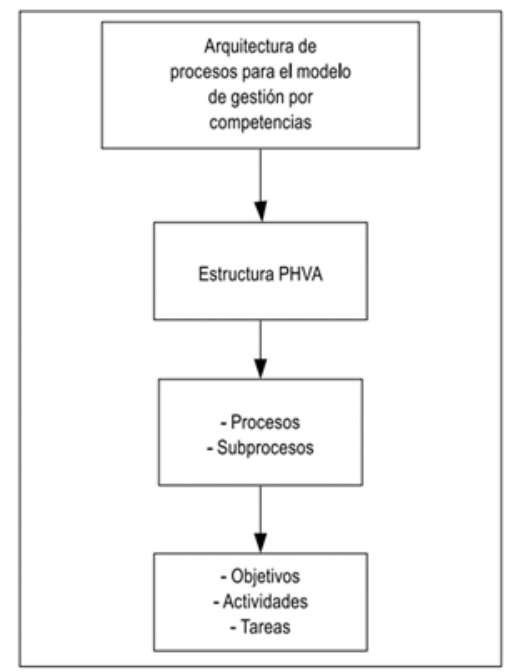

Fuente: (Gómez y Luna, 2013).

Después de estudiar las metodologías aquí mencionadas y con base en ellas, se desarrolló la metodología propuesta en este artículo. El método de elaboración de la misma se presenta en el siguiente apartado.

\section{MÉTODO}

Para el diseño preliminar de la metodología se analizaron los ciclos de vida de: GC de Castillo et al. (2015) y AP de Pesantes y PerezSoltero (2020) para identificar actividades con un nivel de correspondencia o que se complementan entre sí, aprovechar sus similitudes y realizar su integración a la 
metodología propuesta. Este análisis se muestra en la tabla 1. Además, se seleccionaron 2 modelos de base para profundizar en algunas actividades: la metodología BPTrends de Harmon (2014) y el modelo de competencias desarrollado por Gómez y Luna (2013). La primera se utilizó para contextualizar la AP en una metodología ya existente y visualizar la aplicación de su ciclo de vida desde otra perspectiva, de donde se tomaron los elementos que se adecuaban al caso de estudio particular. La segunda se seleccionó por ser una metodología que también busca gestionar el conocimiento a través de una AP la cual también sirvió como guía en el proceso del desarrollo de esta metodología.

Tabla 1

\begin{tabular}{|c|c|c|c|c|c|c|c|}
\hline \multirow{2}{*}{$\begin{array}{c}\text { Gestión del } \\
\text { Conocimiento }\end{array}$} & \multicolumn{7}{|c|}{ Arquitectura de procesos } \\
\hline & 1.Compromiso & 2.Requerimientos & 3.Diseño & 4.Construcción & 5.Validación & 6.Despliegue & 7.Mantenimiento \\
\hline 1. Identificación & $\mathrm{X}$ & $\mathrm{X}$ & & & & & \\
\hline 2. Creación & & & $\mathrm{X}$ & & & & \\
\hline 3. Transferencia & & & $\mathrm{X}$ & & & $\mathrm{X}$ & \\
\hline 4. Almacenamiento & & & & $\mathrm{X}$ & $\mathrm{X}$ & & $\mathrm{X}$ \\
\hline 5. Aplicación & & & & & & $\mathrm{X}$ & \\
\hline 6. Evaluación & & & & & & & $\mathrm{X}$ \\
\hline
\end{tabular}

Fuente: Elaboración propia.

En el ciclo de vida de GC aparece primero la identificación del conocimiento existente en la organización, para lo cual se debe conocer primero cómo trabaja la organización, en AP las primeras 2 metaactividades son la parte donde deben identificarse las partes interesadas y el contexto organizacional en el cual se realizará AP. En la etapa de creación de GC se diseñan las actividades y herramientas de la organización de tal manera que se cree un ambiente favorable para que pueda surgir nuevo conocimiento derivado del conocimiento existente y del funcionamiento diario de la empresa, por su parte en el diseño de AP se especifica el nuevo diseño de los procesos y sus elementos, además de la selección de herramientas en las cuales pueda apoyarse dicho diseño. En la etapa de transferencia de GC se diseñan los canales para compartir el conocimiento de la organización, en AP las fases de diseño y despliegue corresponderían esta fase, en el caso del almacenamiento de GC se construye la base de datos donde el conocimiento quedará resguardado y seguirá disponible para su utilización, por su parte AP tiene como parte de su ciclo de vida la construcción, donde se desarrollan y ajustan los procesos, se realiza la agrupación de estos y su mapeo, la validación que sirve para asegurar que en la construcción se cumplan los objetivos de la organización y el mantenimiento que permite realizar ajustes y mejoras a los procesos. La aplicación del conocimiento es crucial para que la GC sea completada, el objetivo principal de gestionar el conocimiento y de todas sus etapas es que todo el conocimiento existente, adquirido, creado, transferido o almacenado en la organización realmente sea utilizado para beneficio y mejora de la misma, en el caso de AP se debe verificar que su implementación se realice correctamente y que realmente signifique un beneficio para ella. Por último, las etapas de evaluación de GC y mantenimiento de AP van encaminadas al monitoreo de resultados y a la repetición del ciclo para realizar los ajustes necesarios y asegurar una mejora continua en la organización.

Con respecto al soporte de los modelos base seleccionados, la primera fase de la metodología propuesta parte del modelo de Harmon (2014), donde, en su primera fase lo que se requiere es entender el contexto empresarial, se interactúa con las personas para poder definir cuáles son sus objetivos y de esta forma comprender a la organización y poder empezar a definir hacia donde se quiere 
llegar. En el caso del modelo de competencias de Gómez y Luna (2013), la etapa de "Planear" es la que sirve de apoyo en esta primera fase de la metodología propuesta, ya que en ella se realiza un proceso de análisis para saber hacia dónde dirigir la estrategia que se aplicará en su metodología para el caso particular donde se aplique, haciendo un análisis detallado de toda la estructura interna de la organización.

La segunda fase de la metodología propuesta, parte de la tercera meta-actividad del ciclo de vida de AP expuesto por Pesantes y Perez-Soltero (2020). En esta parte de diseño se busca definir la arquitectura del proceso futuro. Además se busca definir una estrategia y realizar la estructura para alinear los procesos a dicha estrategia, considerando los recursos existentes y creando o adquiriendo los recursos necesarios para llevarla a cabo, lo cual se desprende de la segunda fase de la metodología de AP, BPTrends, en la cual también se menciona la importancia de realizar el modelado de los procesos, lo cual se propone dentro de la metodología propuesta. En esta fase también se busca diseñar una correcta GC, lo cual se toma en cuenta en el modelo de competencias en el ciclo de PHVA, en la cual las fases de "Hacer" y "Verificar" engloban una parte de esta fase de la metodología, ya que en ellas se realiza una estructuración de cargos y se elaboran los perfiles de puesto para dichos cargos basados en las competencias necesarias en sus profesionales para la ejecución de su trabajo.

En el desarrollo de esta propuesta se presenta la fase 3 donde se debe construir y ejecutar AP, en el caso del ciclo de vida de AP expuesto anteriormente se muestran dos metaactividades distintas que sirven como referencia para el desarrollo de esta fase que son la construcción y el despliegue. Lo que se pretende en la fase 3 es ejecutar el plan diseñado en la fase 2 , aplicando y coordinando cada una de las actividades y herramientas rediseñadas y creadas, dicha coordinación también forma parte de la metodología BPTrends, donde se establece la gobernanza del proceso y se alinean las capacidades de la organización a los procesos. En el ciclo PHVA de Gómez y Luna (2013), algunas de las actividades de esta fase se incluyen en la fase de "Actuar", ya que en ella se implementa el modelo tomando en cuenta los procesos de sensibilización y capacitación, aunque dicha fase también abarca una parte de nuestra última fase ya que comprende un proceso de mejora continua.

Todos los trabajos mencionados anteriormente, indican la importancia de evaluar y llevar un control de los resultados obtenidos con la implementación de sus propuestas, esto para poder lograr la mejora continua, al convertir cada una de las actividades parte del día a día de las empresas, por ello en la fase 4 de la metodología propuesta se incluyó tanto el mantenimiento de AP como la actualización del conocimiento para que la metodología sea repetida cuantas veces sea necesaria y con los ajustes que vaya requiriendo con el paso del tiempo.

En este diseño preliminar de la metodología propuesta, lo que se busca es combinar y aplicar los principales elementos tanto de AP, como de GC, esto para que al implementar AP se pueda aprovechar la creación de la base y la estructura de los procesos de la empresa para, a la par, gestionar el conocimiento.

\section{RESULTADOS}

Como resultado del análisis de las metodologías expuestas y el objetivo de desarrollar una metodología propia para resolver la problemática presentada en la PYME constructora mencionada anteriormente, se obtuvo el diseño preliminar de una metodología para la gestión del conocimiento basada en una arquitectura de procesos la cual se muestra en la figura 3 , además se describen cada una de sus fases en las que se incluye su objetivo, características y las actividades a desarrollar en cada una de ellas.

\subsection{Fase 1}

El objetivo principal de esta fase es entender el contexto organizacional e 
identificar el conocimiento existente, a continuación se explican estas dos actividades.

Actividad 1: Entender el contexto organizacional. Se pretende realizar un análisis detallado del funcionamiento actual de la empresa, enfocado primeramente en los procesos que se llevan a cabo diariamente, asî como en las personas encargadas de realizar las actividades correspondientes a dichos procesos y los conocimientos necesarios para lograrlo. Para el área de procesos el análisis se divide en tres elementos principales, el análisis de las actividades que conforman los procesos, el análisis de las funciones de cada persona del área donde se aplica la metodología y el análisis de las necesidades que abarca tanto los puntos débiles que los miembros de la organización ya conocen, así como las necesidades $\mathrm{u}$ oportunidades de mejora detectadas a través del análisis de los otros elementos.

Actividad 2: Identificar el conocimiento. En esta actividad se busca identificar el conocimiento que se utiliza para la realización de sus procesos, para ello se pretenden analizar la información, las habilidades y la experiencia que requieren las personas para la ejecución de su trabajo.

Para realizar el levantamiento de toda la información relacionada con las actividades mencionadas, además de la observación directa del funcionamiento de la empresa, se requieren elaborar entrevistas a todas las personas involucradas en los procesos de la organización, además de utilizar formatos de apoyo para la clasificación de dicha información, esto para facilitar el análisis de la misma.

Figura 3. Metodología propuesta.

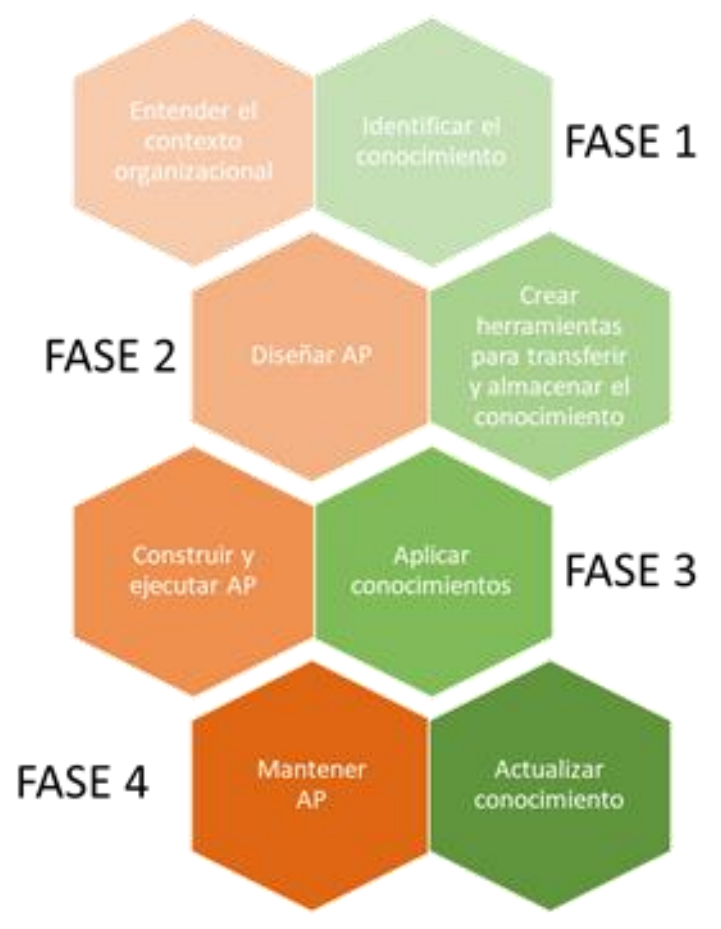

Fuente: Elaboración propia.

\subsection{Fase 2}

El objetivo de esta fase es la planeación y diseño de los procesos y de la organización, así como la creación de herramientas por medio de las cuales se pueda transferir el conocimiento dentro de la empresa y almacenarlo. Estas actividades son explicadas a continuación. 
Actividad 1: Diseñar AP. Se pretende realizar el diseño de una estrategia para lograr hacer más eficientes cada uno de los procesos que se llevan a cabo en el área de aplicación de la metodología, además de diseñar o adaptar las herramientas necesarias para que la empresa realice una correcta gestión del conocimiento en dichos procesos.

En el diseño de procesos se debe definir la estrategia para así poder dar paso al diseño y mejora de los procesos y una vez estructurados se procederá al modelado de estos. Algo importante en esta estrategia es determinar la estructura interna del proceso para soportar la gestión del conocimiento.

Actividad 2: Crear herramientas para transferir y almacenar el conocimiento. Para el diseño de GC, se requiere diseñar el uso que se les dará a las herramientas que se implementen en el rediseño de los procesos, los perfiles de cada puesto de trabajo para que cumplan con los requerimientos de los nuevos procesos y la creación de una base de conocimientos para almacenar y dar uso, de forma ordenada, a todo el conocimiento y la información que ya existe en la organización, así como lo que se va creando conforme pasa el tiempo, esto a través del diseño del manejo de la información $y$ el conocimiento que se desprende diariamente en la ejecución de cada proceso.

\subsection{Fase 3}

El objetivo de esta fase es ejecutar el plan del diseño de procesos, coordinar cada una de las acciones propuestas y aplicar los conocimientos tanto existentes como los que se vayan generando conforme se llevan a cabo los procesos. A continuación de describen las actividades para esta fase.

Actividad 1: Construir y ejecutar AP. Se comunica tanto la estrategia como la nueva estructura y diseño de los procesos, así como las herramientas de GC que se van a utilizar; seguido de esto se asignarán formalmente las actividades para cada persona. Se pretende que cada persona actué de acuerdo con las tareas asignadas y que las nuevas herramientas queden totalmente integradas a los procesos de la organización.
Actividad 2: Aplicar conocimientos. Se capacita a las personas para la correcta utilización de las herramientas seleccionadas en el diseño y se verifica que se implementen correctamente, además se les da acceso a la base de conocimientos creada para que puedan disponer de la información y el conocimiento existente en ella para el desarrollo de sus labores e incluso aportar nueva información y conocimiento a la misma. En esta actividad se les informa y se les hace entrega de cualquier información o documentación adicional que surja en el diseño de cada caso particular y que sea relevante para su uso.

Aunque estos elementos dependen en gran medida de la empresa, se contará con el apoyo y la supervisión del responsable de este proyecto para verificar que efectivamente se realicé la implementación de todos los cambios tal y como se presentan en el diseño. Es muy importante este apoyo ya que el monitoreo realizado en la fase de evaluación y control no va enfocado a la correcta implementación del diseño, si no a los resultados obtenidos realizando correctamente la implementación de la estrategia diseñada para el proyecto.

\subsection{Fase 4}

La finalidad de esta fase es completar los ciclos tanto de arquitectura de procesos como de la gestión del conocimiento. A continuación de describen las actividades para esta fase.

Actividad 1: Mantener AP. Una de las características principales de la AP, dentro del modelado, es la facilidad para realizar cambios estratégicos en los procesos, aun cuando estos están en continuo funcionamiento, por ello es importante llevar un control del comportamiento de los mismos para así lograr ubicar posibles fallas o áreas de oportunidad dentro de los mismos y aprovechar esta característica de AP. Lo que se pretende es evaluar los resultados obtenidos de la ejecución del plan y la aplicación de conocimientos para poder monitorear cada parte, corregir posibles errores y desarrollar 
cada vez una mayor cantidad de mejoras para los procesos de la organización.

Actividad 2: Actualizar conocimientos. Por el lado de GC, es importante dar seguimiento a la forma en que se les da uso a las herramientas implementadas, para poder realizar ajustes en caso de ser necesarios y para ir ampliando la base de conocimientos conforme pasa el tiempo, de la cual a su vez, se debe asegurar un correcto uso y aprovechamiento.

Las fases de la metodología propuesta se pueden realizar cuantas veces sea necesario, ya que se pretende que, además de que permita gestionar el conocimiento existente y el conocimiento que se va creando a través del tiempo en la organización, promueva el control y una constante mejora de sus procesos y operaciones en general, además de una actualización permanente que le permita a la empresa innovar y mantenerse vigente o incluso lograr un crecimiento importante a través de los años.

Aunado a esto, el hecho de que se logren optimizar y hacer más eficientes los procesos de la organización puede traer consigo diferentes beneficios, por ejemplo, reducir los tiempos de ejecución de actividades, lo cual puede repercutir en otro factores como lo son los costos, ya que al optimizar las operaciones diarias de la organización, las personas pueden abarcar un mayor número de actividades lo cual podría provocar la reducción de puestos de trabajo lo que significaría un ahorro para la empresa, o simplemente poder cubrir en un menor tiempo las necesidades de la misma, lo cual puede mejorar la respuesta de los empleados o incluso las relaciones con los clientes.

\section{CONCLUSIONES}

En el estudio de las metodologías de arquitectura de procesos y de gestión del conocimiento revisadas en este trabajo, se observa que todas ellas buscan obtener resultados para el beneficio de las organizaciones, si bien es cierto comparten elementos similares, también hay diferencias en la forma de presentarse y desarrollarse. De ahí surgen las distintas opciones y posibilidades de tomar de cada una de ellas los elementos y las formas de ejecutarlos que mejor se adecuan a cierta problemática de interés. Así que, en el desarrollo de este diseño preliminar de la metodología propuesta, además de tomar elementos de las metodologías estudiadas, se realizaron ajustes que se adaptaron a la forma de trabajar en una PYME constructora.

Finalmente, el objetivo de esta metodología es apoyar a las PYMEs a tener un mejor control de sus procesos de negocio, a identificar, reutilizar y compartir conocimiento de actividades administrativas y técnicas llevadas a cabo por roles de procesos, además facilitará realizar cambios debido a su entorno de trabajo dinámico.

Esta metodología se encuentra en proceso de aplicación, por lo que aún se está obteniendo la evidencia empírica de lo realizado en cada una de las fases. Una vez concluida la implementación, se estará en condiciones de conocer y documentar a detalle los resultados obtenidos con su aplicación, lo cual permitirá verificar que se cumpla con el objetivo aquí expuesto. Por otro lado, se ha observado que conforme se ha ido avanzando en dicha aplicación, le metodología ha sufrido pequeños cambios que la hacen cada vez más flexible, lo que permitirá después de hacer algunas adecuaciones, adaptarse a resolver problemáticas similares en otras organizaciones.

En la actualidad el mundo se encuentra en constante cambio y la tecnología avanza rápidamente, es necesario que las empresas se adapten a dichos cambios, para ello es importante que se invierta en proyectos de investigación que permitan desarrollar propuestas para que las organizaciones puedan subsistir, seguir aprendiendo, innovando $\mathrm{y}$ creciendo continuamente.

La situación de la pandemia que se vive en la actualidad, demuestra la importancia de 
que las organizaciones sean flexibles y se adapten a cualquier cambio. Muchas organizaciones no han logrado sobrepasar esta crisis por diferentes motivos, así que poner en práctica la gestión del conocimiento será de gran ayuda tanto en el ámbito operacional como para lograr atraer nuevos clientes, ya que ante los cambios tan drásticos que está generando dicha situación, lo que queda es innovar. 


\section{REFERENCIAS}

Angulo, R. (2017). Gestión del conocimiento y aprendizaje organizacional: una visión integral. Informes Psicológicos, 17(1), pp. 53-70.

Castillo L., Farías G., Lavín J., \& Pedraza A. (2015). El contador público y la gestión del conocimiento: Un estudio exploratorio. Vincula Tégica Efan, 1(1), pp. 290-313.

Chinowsky, P., \& Carrillo, P. (2007). The knowledge management to learning organizations connection. J. Manage. Eng., 23:3(122), pp. 122-130.

Choo, C. W. (1998). The Knowing Organization: How organizations use information to construct meaning, create knowledge, and make decisions. International Journal of Information Management, 16(5), pp. 329-340.

Dalkir, K. (2017). Knowledge Management in Theory and Practice. Third edition. MIT Press.

Gómez, A., \& Luna, C. (2013). Diseño de una Arquitectura por Proceso para el Modelo de Competencias. Ingeniare, 15, 81.

Harmon, P. (2014). Business process change, 3era. Ed. Morgan Kaufmann.

Harmon, P. (2003). Business Process Change: A Manager's Guide to Improving, Redesigning, and Automating Processes. Morgan Kaufmann.

Hartman, T., \& Fisher, M. (2007). Supporting the constructability review with 3D/4D models. Build. Res. Inform., 35(1), pp. 70-80.

Kazi, A. (2005). Knowledge management in the construction industry: A socio-technical perspective, Idea Group, pp. 0-400.

Kivrak, S., Gokhan, A., Dikmen, I., \& Birgonul, T. (2008). Capturing knowledge in construction projects: Knowledge platform for contractors. J. Manage. Eng., 24:2(87), pp. 87-95.

Marulanda, C., Giraldo, J., \& Serna, H. (2015). Modelo de evaluación de gestión del conocimiento para las pymes del sector de tecnologías de la información. ADminister, 26, pp. 17 - 39.

Omotayo, F. O. (2015). Knowledge Management as an important tool in Organisational Management: A Review of Literature. Library Philosophy and Practice (e-journal). 1238.

Pesantes, M., Lemus, C., Mitre, H. A., \& Mejia, J. (2012). Software process architecture: Roadmap. Proceedings-2012 9th Electronics, Robotics and Automotive Mechanics Conference, pp. 111116.

Pesantes, M., \& Perez-Soltero, A. (2020). Developing an implementation plan for a domain specific process architecture in a multimodel environment. Proceedings of World Research Society International Conference, pp. 62-69.

Portela, F., Santos, M., Machado, J., Abelha, A., Silva, Á., \& Rua, F. (2014). Pervasive and intelligent decision support in intensive medicine - The complete picture. Lecture Notes in Computer Science (Including Subseries Lecture Notes in Artificial Intelligence and Lecture Notes in Bioinformatics), 8649 LNCS, pp. 87-102.

Schwartz, D. (2006). Encyclopedia of knowledge management, Idea Group.

Shelbourn, M., Bouchlaghem, D., Anumba, C., Carrillo, P., Khalfan, M., \& Glass, J. (2006). Managing knowledge in the context of sustainable construction. Journal of Information Technology in Construction, 11, pp. 57-71.

Szelągowski, M., \& Berniak-Woźny, J. (2019). The knowledge and process continuum. Knowledge and Process Management, 26(4), pp. 308-320. 